\title{
NON-LOCAL SIMILARITY BASED TENSOR DECOMPOSITION FOR HYPERSPECTRAL IMAGE DENOISING
}

\author{
Fan Xu' ${ }^{1}$ Xiao Bai ${ }^{1}$, Jun Zhou ${ }^{2}$ \\ ${ }^{1}$ School of Computer Science and Engineering, Beihang University, Beijing, China \\ ${ }^{2}$ School of Information and Communication Technology, Griffith University, Nathan, Australia
}

\begin{abstract}
Compared to traditional color or grayscale images, hyperspectral image (HSI) can help deliver more faithful representation of ground objects and enhance the performance of many computer vision tasks. However, an HSI is often corrupted by various noises, which has serious impact on the subsequent processing. Considering the non-local similarity across spatial domain and global similarity along spectral domain, a novel denoising method based on tensor decomposition is proposed in this paper. Firstly, 3D full band patches extracted from the HSI are grouped to form a 4th-order tensor by utilizing the non-local similarity in a proper window size. Then the task of hyperspectral image denoising is transformed into a high order tensor approximation problem, which can be efficiently solved by alternating optimization. An iterative denoising strategy is adopted for better effect in practice. Experimental results on simulated and real HSI data show that the proposed algorithm outperforms several stateof-the-art methods.
\end{abstract}

Index Terms - Hyperspectral Image, Denoising, Nonlocal Similarity, Tensor Decomposition

\section{INTRODUCTION}

Hyperspectral image (HSI) can be thought as a set of 2D spatially organized pixels where each pixel contains an entire spectral distribution over light wavelengths. Hyperspectral image has led to numerous applications in remote sensing [1], classification [2,3] and detection [4,5], and more. However, conventional hyperspectral imaging suffers from issues such as limited illumination in individual band and shorting sensing time which introduce noises into the image processing. Therefore, HSI denoising is a critical step to improve the quality of HSI.

Aiming at the aforementioned issues, we propose a novel denoising method by considering both non-local similarity across the spatial domain and global correlation among the spectral domain. On one hand, a natural scene contains a collection of similar local patches over the space, composing of homologous aggregation of microstructures. On the other hand, an HSI contains a large amount of spectral redundancy.
It means that images obtained over multiple bands are highly correlated. And it has been extensively shown that such two kinds of prior knowledge are very helpful for various HSI recovery problems [6-10].

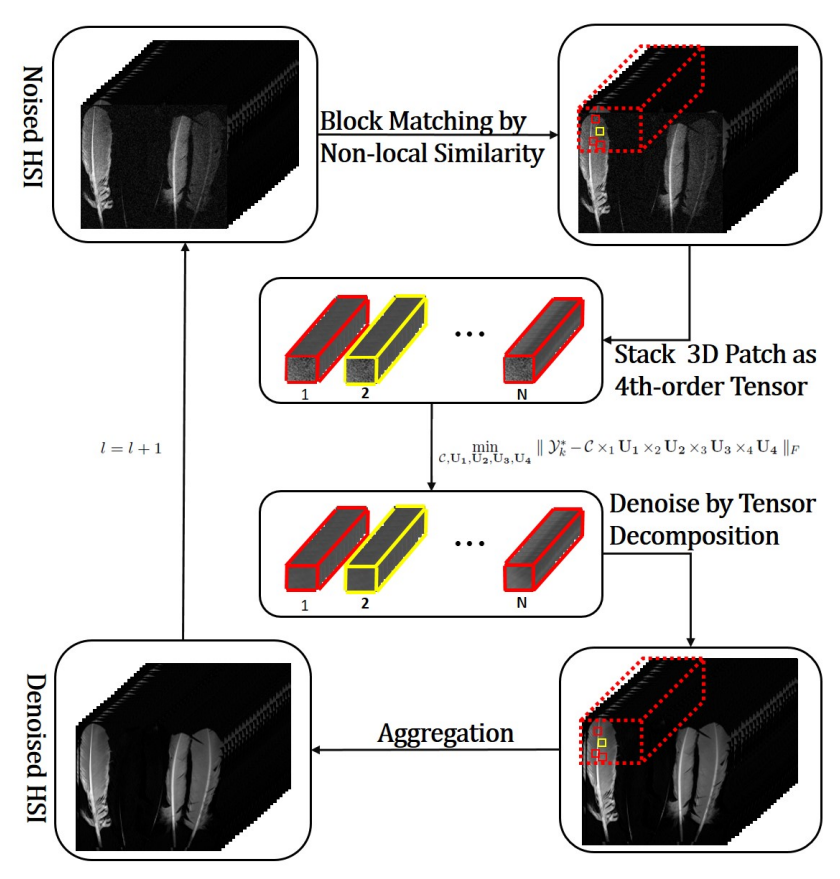

Fig. 1. Overview of the proposed HSI denoising method.

In this paper, tensor decomposition based HSI denoising technique is adopted in our model, which not only emphasizes the similarity among non-local full band patches but also utilizes the redundancy of spectral space. Specifically, we treat each full band patch as a 3rd-order tensor with two spatial modes and a spectral mode, and then build a 4th-order tensor by stacking all these non-local similar full band patches (shown at Fig.1). Then the denoising problem can be transferred into tensor recovery by utilizing the inherent spatial and spectral correlation of the full band patch groups. We solve the tensor recovery problem by implementing the alternative optimization of tensor decomposition and then aggregate the 
denoised 3D patches in the clean HSI iteratively.

\section{RELATED WORKS}

2D extended approach: Over the past several decades, many 2D image denoising methods have been developed, such as NLM [11], K-SVD [12], and BM3D [13]. These methods can be extended into HSI denoising problem by considering the images located at different bands separately. The band-wised method, however, ignores the intrinsic properties of HSI and cannot achieve superior effect in normal conditions. Another more reasonable extension is specifically designed for patchbased image denoising methods, which takes the small local patches into consideration. Instead of processing 2D patches on traditional image, 3D denoising method can be developing by processing small 3D cubes on both spatial and spectral domains. The 3D cube based approach is represented by a BM4D [14] method, which utilizes the 3D non-local similarity of HSI to remove noises in similar 3D cubes collaboratively. Nevertheless, these methods have not taken the property of high correlation among spectral into account and still have much room to improve.

Tensor based approach: An HSI can be regarded as a stack of 2D gray images, which can be seen as a 3rd-order tensor. Accordingly, the low-rank approximation (LRTA) [15] utilizes the Tucker decomposition [16] of the input HSI. Li$\mathrm{u}$ et al. designed a PARAFAC $[17,18]$ method by employing parallel factor analysis. Though both approaches take the correlation over different bands into consideration and try to eliminate the spectral redundancy of HSIs, they ignore the non-local similarity of the full band patches across the spatial domain. Tensor based method is currently represented by tensor dictionary learning (TDL) [19] which takes the HSI properties into account. However, clustering for full band patches cannot make full use of the fine grained non-local similarity structures, and still have not reached the full potential for handling the denoising task.

\section{HYPERSPECTRAL IMAGE DENOISING MODEL}

A noise free HSI $\mathcal{X}$ degraded by additive zero mean Gaussian noise can be modeled as $\mathcal{Y}=\mathcal{X}+\mathcal{N}$, where $\mathcal{N}$ is the noise with a known variance $\sigma^{2}$ and zero mean. The ultimate goal of denoising is to find a good estimate $\widehat{\mathcal{X}}$ of clean HSI $\mathcal{X}$ from noisy $\mathcal{Y}$.

\subsection{Non-local Similarity Patch Matching}

General non-local similarity based denoising framework has been widely applied in traditional image denoising. The similar denoising model can be extended to HSI cases. Denote $n_{h}, n_{w}$ and $n_{s}$ as the height and width in the spatial dimension and number of spectral band of an input HSI respectively, then we can express the HSI as a 3 rd-order tensor $\mathcal{Y} \in \mathbb{R}^{n_{h} \times n_{w} \times n_{s}}$ with two spatial modes and one spectral mode. By sweeping across the HSI with overlaps, we can build a group of 3D full band patches $\left\{P_{i, j}\right\}_{1 \leq i \leq n_{h}-n_{p}, 1 \leq j \leq n_{w}-n_{p}} \subset \mathbb{R}^{n_{p} \times n_{p} \times n_{s}}$ to represent the HSI, where each band of a full band patch is ordered lexicographically as a column vector. We can now reformulate all full band patches as a group of 3D patches $\Phi_{\mathcal{Y}}=\left\{\mathcal{Y}_{i} \in \mathbb{R}^{n_{p} \times n_{p} \times n_{s}}\right\}_{i=1}^{N}$, where $N=\left(n_{h}-n_{p}+1\right) \times$ $\left(n_{w}-n_{p}+1\right)$ is the number of patches over the whole $\mathrm{H}$ SI.

For a given local full band patch $P_{r e f}$, we can find a collection of full band patches $P_{k}$ similar to it from a predefined searching window by utilizing the non-local similarity matching, where $k \in[1, K]$ and $K$ is the number of similar patches. The similarity between $P_{r e f}$ and $P_{k}$ can be formulated as:

$$
\operatorname{Sim}\left(P_{\text {ref }}, P_{k}\right)=\sqrt{\left\|P_{\text {ref }}-P_{k}\right\|^{2}}
$$

The smaller the $\operatorname{Sim}\left(P_{r e f}, P_{k}\right)$, the more similar the two patches may turn out to be. Denote $\mathcal{Y}_{k}^{*} \in \mathbb{R}^{n_{p} \times n_{p} \times n_{s} \times n_{K}}$ as the 4th-order tensor stacked by $P_{r e f}$ and its non-local similar full band patches $P_{k}$ in search window. Then we can estimate the corresponding true non-local similarity full band patches $\mathcal{X}_{k}^{*}$ from its corrupted $\mathcal{Y}_{k}^{*}$ by solving the following optimization problem:

$$
\min _{\mathcal{X}_{k}^{*}}\left\|\mathcal{Y}_{k}^{*}-\mathcal{X}_{k}^{*}\right\|_{F}
$$

\subsection{4th-Order Tensor Decomposition}

According to the tensor decomposition theory, the aforementioned optimization problem can be transformed into the following problem:

$$
\begin{array}{ll} 
& \min _{\mathcal{C}, \mathbf{U}_{\mathbf{1}}, \mathbf{U}_{\mathbf{2}}, \mathbf{U}_{\mathbf{3}}, \mathbf{U}_{\mathbf{4}}}\left\|\mathcal{Y}_{k}^{*}-\mathcal{X}_{k}^{*}\right\|_{F} \\
\text { s.t. } & \mathcal{X}_{k}^{*}=\mathcal{C} \times{ }_{1} \mathbf{U}_{\mathbf{1}} \times_{2} \mathbf{U}_{\mathbf{2}} \times_{3} \mathbf{U}_{\mathbf{3}} \times_{4} \mathbf{U}_{\mathbf{4}} \\
& \mathbf{U}_{\mathbf{n}}^{\mathbf{T}} \mathbf{U}_{\mathbf{n}}=\mathbf{I}, n=1,2,3,4
\end{array}
$$

where $\mathbf{U}_{\mathbf{1}} \in \mathbb{R}^{n_{p} \times r_{p}}, \mathbf{U}_{\mathbf{2}} \in \mathbb{R}^{n_{p} \times r_{p^{\prime}}}, \mathbf{U}_{\mathbf{3}} \in \mathbb{R}^{n_{S} \times r_{S}}, \mathbf{U}_{\mathbf{4}} \in$ $\mathbb{R}^{n_{K} \times r_{K}}$ correspond the basis vectors in the four modes of $\mathcal{Y}^{*}$ with $n_{p} \geq r_{p}, n_{p} \geq r_{p^{\prime}}, n_{S}>r_{S}$ and $n_{K} \geq r_{K}$. Here $\mathcal{C} \in \mathbb{R}^{r_{p} \times r_{p^{\prime}} \times r_{S} \times r_{K}}$ is the so-called core tensor and $r_{S}<n_{S}$ leads to the dimensionality reduction in the spectral mode of $\mathcal{Y}_{k}^{*}$. The AIC/MDL criterion [20] can be used to determine the rank for our tensor model. More specifically, Eq.(3) could be readily solved by the Tucker decomposition [16] technique. By applying the alternate solution, when $n=1$, the original problem can be break up into the following subproblem:

$$
\begin{array}{ll}
\min & \left\|\mathbf{U}_{1}^{T} \mathbf{W}\right\|_{F} \\
\text { s.t. } & \mathbf{W}=\mathbf{Y}_{1}\left(\mathbf{U}_{4} \otimes \mathbf{U}_{3} \otimes \mathbf{U}_{2}\right)
\end{array}
$$

where $\mathbf{Y}_{n}$ is the flattened of $\mathcal{Y}$ at mode-n and $\otimes$ is the Kronecker products. It is not difficult to derive the other conditions when $n=2,3,4$. 


\subsection{Iterative Denoising Optimization}

Generally, denoising once is not enough to get the desired denoised result in practice. Therefore, we designed an iterative denoising strategy for the proposed method, which is verified to be a process to take the trade-off between bias and variance.

$$
\mathcal{Y}^{(l)}=\mathcal{X}^{(l-1)}+\delta\left(\mathcal{Y}-\mathcal{X}^{(l-1)}\right)
$$

where $l$ is the number of iterations, $\delta \in(0,1)$ is the residual proportionality coefficient. By utilizing Eq. (5), the denoised effect could be optimized. The maximum iteration number $L$ should be properly selected to ensure the optimization result.

The proposed algorithm for HSI denoising can be summarized in Algorithm 1.

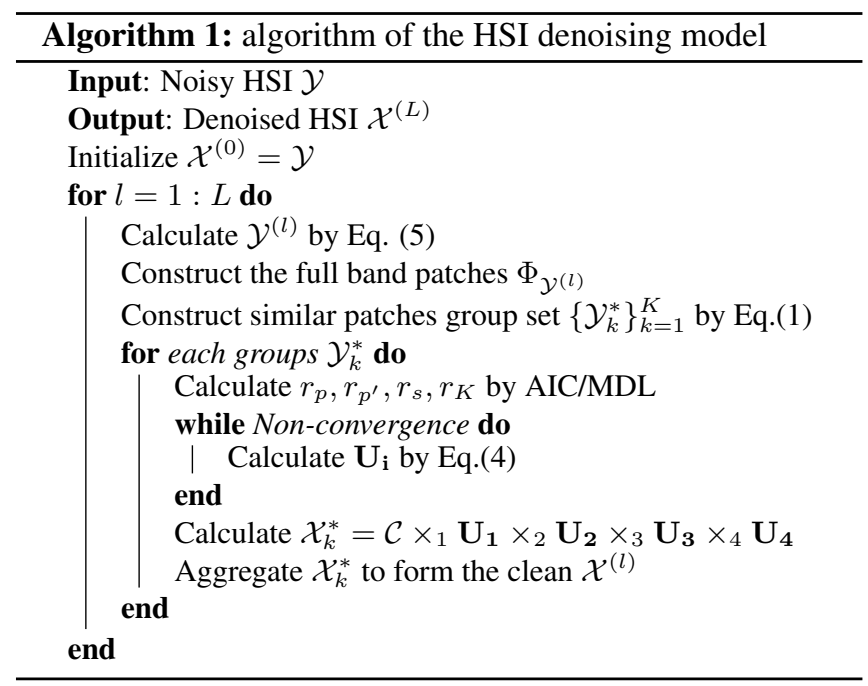

\section{EXPERIMENTAL RESULTS}

To demonstrate the effectiveness of the proposed method, we compare it with 8 recently developed denoising methods on both Columbia Dataset and Pavia University Dataset.

\subsection{Simulated HSI denoising}

The Columbia Dataset [22] contains a wide variety of realworld materials and objects, which include 32 real-world scenes. Every HSI includes full spectral resolution reflectance data collected from $400 \mathrm{~nm}$ to $700 \mathrm{~nm}$ in $10 \mathrm{~nm}$ steps with spatial resolution $512 \times 512$ and number of spectral bands 31. The comparison methods include: state-of-the-art for the tensor-based approach, LRTA [15], PARAFAC [17], and TDL [19]; 2D extended band-wise approach, band-wise KSVD [21] and band-wise BM3D [13]; current method for the 2D extended 3D-cube-based approach, K-SVD [12] and BM4D [14]. To evaluate the performance of the denoising methods, picture quality indices (PQI), e.g. the peak signalto-noise ratio (PSNR), structure similarity (SSIM) and feature similarity (FSIM) are used to measure the similarity of denoised HSI and the fully sampled HSI which is assumed to be ground truth.

We corrupt the clean image with an additive zero mean Gaussian noises with $\sigma$ ranging from 20 to 60 . The residual proportionality coefficient $\delta$ is set to 0.1 and the maximum iteration number $L$ is determined automatically according to the $\sigma$. The size of of searching window full band patches is set 50 pixels. For each noise parameter [12], all the three PQI values for each competing HSI denoising methods on all 32 scenes have been calculated and recorded. Table. 1 lists the average performance of all methods. From these comparison, the advantage of the proposed method can be evidently observed. We show in Fig. 2 at band $620 \mathrm{~nm}$ with $200 \times 200$ window size extracted from stuffed toy, cloth and feather for further testification of our method. Method K-SVD, BM4D, TDL are selected to be shown and it's obvious that the proposed algorithm outperforms the comparing method.

\subsection{Real HSI denoising}

In this section, Pavia University dataset of natural scene is used in our experiments. The original HSI is of the size $610 \times 340 \times 103$. For the purpose of calculating convenience and overcoming redundancy, we extract 31 bands and leave the remaining test data with a size of $610 \times 340 \times 31$. We adopts the similar implementation strategies and parameter settings for all competing methods as previous simulated experiments.

We illustrate the experimental results at the 5th band of the HSI in Fig.3. It can be easily observed that the image restored by our method is capable of properly removing the Gaussian noise while finely preserving the structure underlying the HSI.

\section{CONCLUSION}

In this paper, a new denoising model has been proposed based on tensor decomposition for hyperspectral image which utilizes the non-local similarity across spatial space efficiently. We have also solved this model by optimization algorithm iteratively. The method has been shown to consistently achieve superior denoising result, on both simulated and real HSI, than the state-of-the-art methods.

\section{ACKNOWLEDGMENT}

This work is supported by NSFC project No.61370123 and BNSF project No.4162037. It is also supported by funding from State Key Lab of Software Development Environment in Beihang University. 


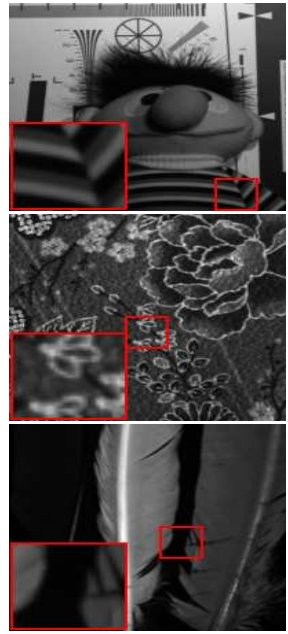

(a) Original

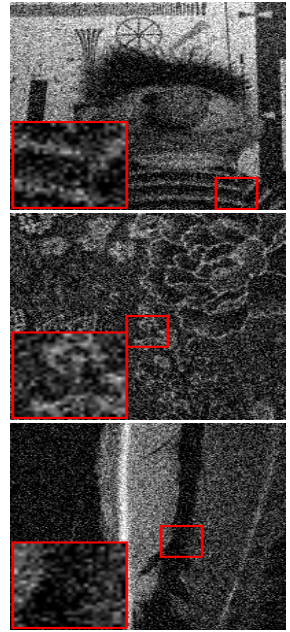

(b) Noisy

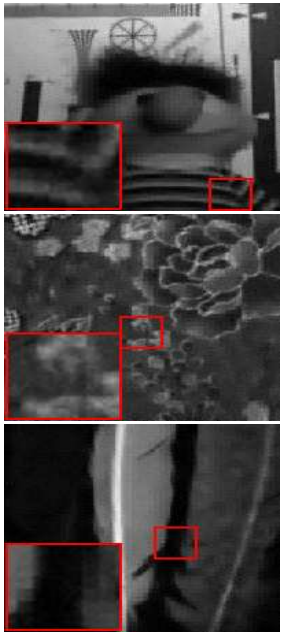

(c) K-SVD

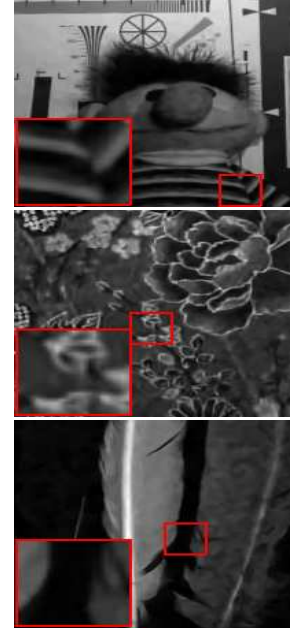

(d) BM4D

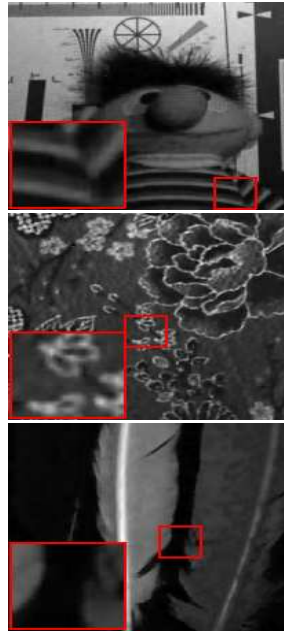

(e) TDL

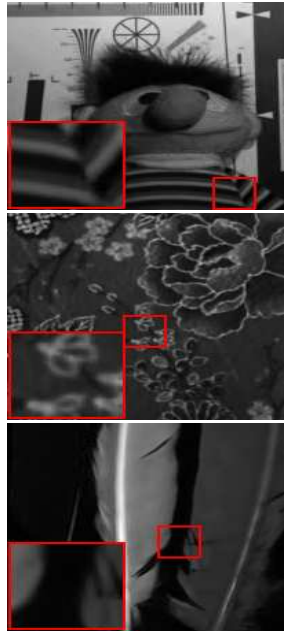

(f) Ours

Fig. 2. (a) The images at band 620nm of stuffed toy, cloth and feather; (b) The corresponding images corrupted by the mixture of $\sigma=40$ Gaussian noise; (c)-(f) The restored images obtained by 4 of the comparing 8 denoising methods. The demarcated areas in each image is amplified at a 4 times larger scale for easy observation of details.

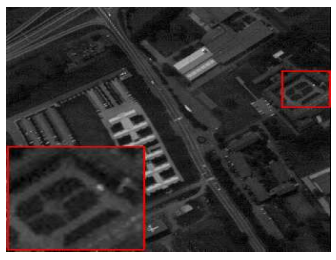

(a) Original

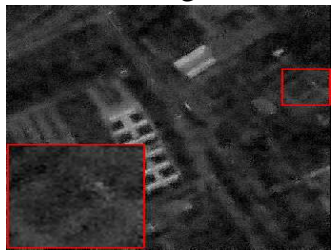

(f) BwK-SVD

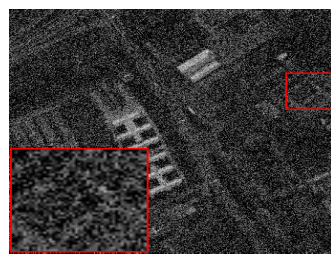

(b) Noisy

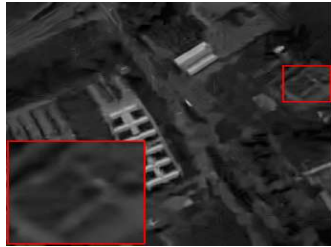

(g) BwBM3D

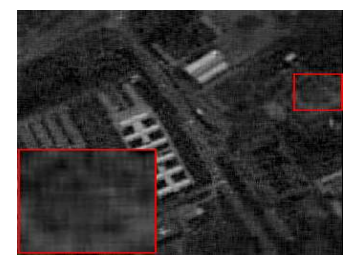

(c) LRTA

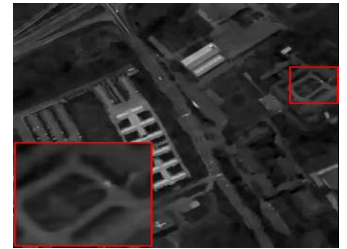

(h) BM4D

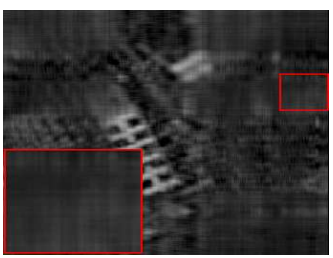

(d) PARAFAC

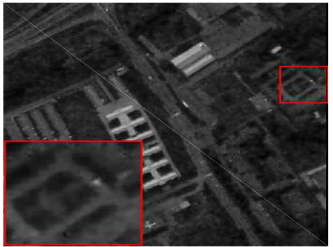

(i) TDL

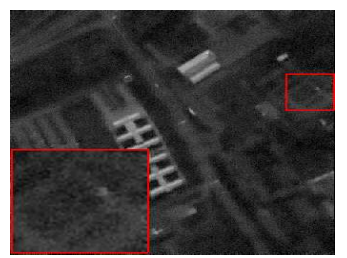

(e) KSVD

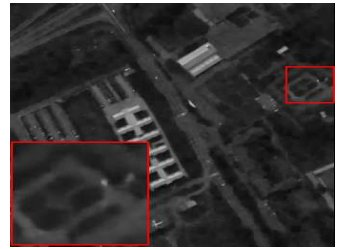

(j) Ours

Fig. 3. (a) The Original image located at the $5^{s t}$ band in Pavia University dataset; (b) The corrupted image by Gaussian noise of $\sigma=40$; (c)-(j) The denoised HSI obtained by the comparing denoising method. The demarcated areas in each image are amplified at a 4 times larger scale.

\begin{tabular}{|c|c|c|c|c|c|c|c|c|c|}
\hline \multirow{2}{*}{ Methods } & \multicolumn{3}{|c|}{$\sigma=20$} & \multicolumn{3}{c|}{$\sigma=40$} & \multicolumn{3}{c|}{$\sigma=60$} \\
\cline { 2 - 10 } & PSNR & SSIM & FSIM & PSNR & SSIM & FSIM & PSNR & SSIM & FSIM \\
\hline Noisy & 17.59 & 0.2433 & 0.6253 & 14.27 & 0.1870 & 0.5254 & 11.38 & 0.0962 & 0.4174 \\
PARAFAC [17] & 35.36 & 0.8932 & 0.9599 & 31.52 & 0.7609 & 0.9246 & 29.30 & 0.7857 & 0.8775 \\
LRTA [15] & 37.67 & 0.9437 & 0.9722 & 34.28 & 0.8212 & 0.9223 & 31.53 & 0.8036 & 0.9028 \\
BwK-SVD [21] & 33.95 & 0.8002 & 0.9603 & 29.65 & 0.7157 & 0.9200 & 27.89 & 0.7079 & 0.8564 \\
K-SVD [12] & 36.02 & 0.9397 & 0.9721 & 33.41 & 0.7285 & 0.9203 & 31.90 & 0.8110 & 0.9123 \\
BwBM3D [13] & 38.21 & 0.9497 & 0.9747 & 34.94 & 0.8802 & 0.9358 & 33.84 & 0.8761 & 0.9251 \\
BM4D [14] & 40.57 & 0.9665 & 0.9878 & 37.79 & 0.9105 & 0.9583 & 34.62 & 0.8984 & 0.9399 \\
TDL [19] & 40.96 & 0.9707 & 0.9813 & 38.16 & 0.9334 & 0.9716 & 35.41 & 0.9012 & 0.9421 \\
Ours & $\mathbf{4 2 . 8 7}$ & $\mathbf{0 . 9 8 9 4}$ & $\mathbf{0 . 9 9 4 6}$ & $\mathbf{3 9 . 8 5}$ & $\mathbf{0 . 9 4 7 2}$ & $\mathbf{0 . 9 7 6 0}$ & $\mathbf{3 6 . 2 6}$ & $\mathbf{0 . 9 0 4 8}$ & $\mathbf{0 . 9 5 6 4}$ \\
\hline
\end{tabular}

Table 1. Average performance of 8 competing method with respect to the different additive noise. The results are obtained by averaging through the 32 scenes and the varied parameters. 


\section{REFERENCES}

[1] U. Heiden, K. Segl, S. Roessner, and H. Kaufmann, "Determination of robust spectral features for identification of urban surface materials in hyperspectral remote sensing data," Remote Sensing of Environment, vol. 111, no. 4, pp. 537-552, 2007.

[2] M. Fauvel, J.A. Tarabalka, Y.and Benediktsson, J. Chanussot, and J. C. Tilton, "Advances in spectralspatial classification of hyperspectral images," Proceedings of the IEEE, vol. 101, no. 3, pp. 652-675, 2013.

[3] S. Liu and X. Bai, Discriminative features for image classification and retrieval, Elsevier Science Inc., 2012.

[4] X. Bai, H. Zhang, and J. Zhou, "Vhr object detection based on structural feature extraction and query expansion," IEEE Transactions on Geoscience and Remote Sensing, vol. 52, no. 10, pp. 6508-6520, 2014.

[5] N. M. Nasrabadi, "Hyperspectral target detection : An overview of current and future challenges," IEEE Signal Processing Magazine, vol. 31, no. 1, pp. 34-44, 2014.

[6] W. Cao, Y. Wang, C. Yang, X. Chang, Z. Han, and Z. X$\mathrm{u}$, "Folded-concave penalization approaches to tensor completion," Neurocomputing, vol. 152, no. C, pp. 261273, 2015.

[7] X. L. Zhao, F. Wang, T. Z. Huang, and M. K. Ng, "Deblurring and sparse unmixing for hyperspectral images," IEEE Transactions on Geoscience and Remote Sensing, vol. 51, no. 7, pp. 4045-4058, 2013.

[8] R. Kawakami, Y. Matsushita, J. Wright, and M. BenEzra, "High-resolution hyperspectral imaging via matrix factorization," in Computer Vision and Pattern Recognition, 2011, pp. 2329-2336.

[9] M. Ye, Y. Qian, and J. Zhou, "Multitask sparse nonnegative matrix factorization for joint spectralcspatial hyperspectral imagery denoising," IEEE Transactions on Geoscience and Remote Sensing, vol. 53, no. 5, pp. 2621-2639, 2015.

[10] X. Bai, Z. Guo, Y. Wang, Z. Zhang, and J. Zhou, "Semisupervised hyperspectral band selection via spectralcspatial hypergraph model," IEEE Journal of Selected Topics in Applied Earth Observations and Remote Sensing, vol. 8, no. 6, pp. 2774-2783, 2015.

[11] A. Buades, B. Coll, and J. M. Morel, "A non-local algorithm for image denoising," in IEEE Computer Society Conference on Computer Vision and Pattern Recognition, 2005, pp. 60-65.
[12] M. Elad and M. Aharon, "Image denoising via learned dictionaries and sparse representation," in Computer Vision and Pattern Recognition, 2006 IEEE Computer Society Conference on, 2006, pp. 895-900.

[13] K. Dabov, A. Foi, V. Katkovnik, and K. Egiazarian, "Image denoising by sparse 3-d transform-domain collaborative filtering.," IEEE Transactions on Image Processing A Publication of the IEEE Signal Processing Society, vol. 16, no. 8, pp. 2080-2095, 2007.

[14] A. Foi, "Nonlocal transform-domain denoising of volumetric data with groupwise adaptive variance estimation," vol. 8296, no. 3, pp. -, 2012.

[15] N. Renard, S. Bourennane, and J. Blanc-Talon, "Denoising and dimensionality reduction using multilinear tools for hyperspectral images," IEEE Geoscience and Remote Sensing Letters, vol. 5, no. 2, pp. 138-142, 2008.

[16] T. G. Kolda and B. W. Bader, "Tensor decompositions and applications," Siam Review, vol. 51, no. 3, pp. 455500, 2009.

[17] Xuefeng Liu, Salah Bourennane, and Caroline Fossati, "Denoising of hyperspectral images using the parafac model and statistical performance analysis," IEEE Transactions on Geoscience and Remote Sensing, vol. 50, no. 10, pp. 3717-3724, 2012.

[18] X. Guo, X. Huang, L. Zhang, and L. Zhang, "Hyperspectral image noise reduction based on rank-1 tensor decomposition," Isprs Journal of Photogrammetry and Remote Sensing, vol. 83, no. 9, pp. 50-63, 2013.

[19] Y. Peng, De. Meng, Z. Xu, C. Gao, Y. Yang, and B. Zhang, "Decomposable nonlocal tensor dictionary learning for multispectral image denoising," pp. 29492956, 2014.

[20] M. Wax and T. Kailath, "Detection of signals by information theoretic criteria," IEEE Transactions on Acoustics Speech and Signal Processing, vol. 33, no. 2, pp. 387-392, 1985.

[21] M. Aharon, M. Elad, and A. Bruckstein, "K-svd: An algorithm for designing overcomplete dictionaries for sparse representation," IEEE Transactions on Signal Processing, vol. 54, no. 11, pp. 4311-4322, 2006.

[22] F. Yasuma, T. Mitsunaga, D. Iso, and S. K. Nayar, "Generalized assorted pixel camera: postcapture control of resolution, dynamic range, and spectrum.," IEEE Transactions on Image Processing A Publication of the IEEE Signal Processing Society, vol. 19, no. 9, pp. 2241, 2010. 